\title{
Diagnósticos de enfermagem em pacientes com úlcera venosa crônica: estudo observacional
}

\section{Nursing diagnoses in patients with chronic venous ulcer: observational study}

Glycia de Almeida Nogueira ${ }^{1}$, Beatriz Guitton Renaud Baptista Oliveira², Rosimere Ferreira Santana ${ }^{3}$, Ana Carla Dantas Cavalcanti ${ }^{4}$

\footnotetext{
${ }^{1}$ Enfermeira. Discente do Programa de Pós-Graduação em Ciências do Cuidado em Saúde e Enfermagem, nível Mestrado, da Universidade Federal Fluminense (UFF). Rio de Janeiro, RJ, Brasil. E-mail: glycianog@yahoo.com.br.

${ }^{2}$ Enfermeira, Doutora em Enfermagem. Professor Titular da Escola de Enfermagem Aurora de Afonso Costa (EEAAC) da UFF. Rio de Janeiro, RJ, Brasil. E-mail: beatrizguitton@globo.com.

${ }^{3}$ Enfermeira, Doutora em Enfermagem. Professor Adjunto da EEAAC/UFF. Rio de Janeiro, RJ, Brasil. E-mail: rosifesa@enf.uff.br.

${ }^{4}$ Enfermeira, Doutora em Enfermagem. Professor Adjunto da EEAAC/UFF. Rio de Janeiro, RJ, Brasil. E-mail: ana_carladc@yahoo.com.br.
}

\section{RESUMO}

Este estudo teve como objetivo analisar os diagnósticos de enfermagem em pessoas com úlcera venosa crônica. Pesquisa observacional, descritiva, de abordagem quantitativa realizado em um ambulatório especializado no tratamento de feridas, com amostra não-probabilística de 20 pacientes. A coleta de dados foi feita no formulário institucional denominado Protocolo de Avaliação dos Clientes com Lesões Tissulares. Os diagnósticos foram estabelecidos por consenso entre quatro pesquisadores com experiência em diagnósticos de enfermagem e tratamento de feridas. Da análise dos dados identificaram-se 16 diagnósticos, sendo que $100 \%$ dos participantes apresentaram: Integridade tissular prejudicada, Perfusão tissular periférica ineficaz, Risco de infecção, Mobilidade física prejudicada e Autocontrole ineficaz da saúde. Estes diagnósticos encontram-se nos domínios Segurança/Proteção, Atividade/Repouso e Promoção da Saúde, que do ponto de vista da prática clínica devem ser focos prioritários na intervenção e avaliação de enfermagem.

Descritores: Úlcera Varicosa; Diagnóstico de Enfermagem; Cuidados de Enfermagem.

\section{ABSTRACT}

This study aimed to analyze nursing diagnoses in people with chronic venous ulcer. An observational, descriptive, quantitative research conducted in an ambulatory specialized in wound treatment, with a non-probabilistic sample of 20 patients. Data collection was performed in an institutional form denominated Assessment Protocol for Clients with Tissue Lesions. Diagnoses were established by consensus among four researchers with experience in nursing diagnoses and wound treatments. From data analysis, 16 diagnoses were identified, with $100 \%$ of participants presenting: Impaired tissue integrity, Ineffective peripheral tissue perfusion, Risk of infection, Impaired physical mobility and Ineffective health self-control. These diagnoses are found in Safety/Protection, Activity/Rest and Health promotion domains, which from the clinical practice stand point should be priority focuses in nursing intervention and assessment. Descriptors: Úlcera Varicosa; Diagnóstico de Enfermagem; Cuidados de Enfermagem. 


\section{INTRODUÇÃO}

As úlceras venosas constituem um sério problema de saúde pública, responsáveis por considerável impacto econômico aos sistemas de saúdes e determinantes de sofrimento e isolamento social, bem como, provocando absenteísmo e desemprego ${ }^{(1-3)}$.

A prevalência de úlceras venosas varia entre os estudos, devido à heterogeneidade dos métodos diagnósticos e as características epidemiológicas da amostra. No Brasil, são descritas prevalências de úlceras venosas ativas e/ou curadas em quase $3,6 \%$ entre indivíduos com idades acima de 15 anos, aumentando entre os mais idosos ${ }^{(4)}$.

A úlcera venosa representa o estágio mais avançado da insuficiência venosa crônica que, por sua vez, está associada à disfunção da bomba muscular da panturrilha e, consequentemente, a hipertensão venosa. Essa bomba muscular é o mecanismo primário para o retorno do sangue dos membros inferiores para o coração, sendo formada pelos músculos da panturrilha, sistema venoso profundo, sistema venoso superficial e sistema das veias perfurantes/comunicantes ${ }^{(4)}$.

Um dos objetivos da enfermagem ao prestar cuidados aos pacientes com úlceras de perna é sistematizar a assistência, com levantamento dos diagnósticos a fim de planejar as intervenções e avaliar a qualidade dos cuidados prestados ${ }^{(5)}$.

A aplicação dos diagnósticos de enfermagem da NANDA-I possibilitam a identificação dos problemas do paciente com vistas ao restabelecimento e a promoção da saúde ${ }^{(6)}$. Por isso, são fundamentais para uma prática assistencial organizada, já que, a partir deles são planejados e realizados cuidados de enfermagem que determinarão resultados de melhora da saúde dos pacientes. A avaliação desses resultados dá visibilidade e corrobora a relevância da equipe de enfermagem nos cuidados à saúde.

No Brasil há poucos estudos a respeito dos diagnósticos de enfermagem em pacientes com feridas, e, mesmo os já publicados, tratam apenas de diagnósticos específicos, como Integridade de pele prejudicada, Integridade tissular prejudicada e Risco de integridade da pele prejudicada ${ }^{(7-9)}$. Ou seja, os diagnósticos não são identificados a partir da integralidade no cuidado, abordando as demais necessidades de cuidado que esses pacientes possam ter, além daquelas relacionadas à pele. Apenas um estudo foi encontrado nesse sentido, porém, restringe-se a pacientes do sexo feminino ${ }^{(10)}$. Por isso, torna-se fundamental a produção de conhecimentos referente os diagnósticos de enfermagem em pacientes com feridas crônicas.

Dessa forma, o objetivo do estudo foi analisar os diagnósticos de enfermagem em pessoas com úlcera venosa crônica.

\section{MÉTODO}

Trata-se de um estudo observacional, descritivo, de abordagem quantitativa, realizado em um ambulatório especializado no tratamento de feridas de um hospital universitário.

A amostra foi não-probabilística, estabelecida conforme o fluxo de atendimento do ambulatório, entre os meses de julho e agosto de 2012, formada por 20 pacientes com feridas de etiologia venosa. Os critérios de inclusão foram: presença de úlcera venosa; idade acima de 18 anos e comparecimento a cinco ou mais consultas de enfermagem no ambulatório. Como critérios de exclusão considerou-se: paciente com distúrbio psiquiátrico e pacientes grávidas.

O instrumento de coleta de dados, denominado Protocolo de Avaliação dos Clientes com Lesões Tissulares, é institucionalizado e constituído de três partes: identificação do paciente (dados sociodemográficos), exame clínico e específico da lesão. Este formulário foi analisado, quanto à aparência e ao conteúdo, por três avaliadores especialistas (enfermeiros com experiência em assistência a pacientes com feridas e em diagnósticos de enfermagem) que sugeriram a inclusão das avaliações da temperatura ao redor da lesão 
e da perfusão capilar, necessárias a um julgamento clínico mais acurado.

Após a adequação do instrumento, os dados de anamnese foram coletados pela pesquisadora nas consultas de enfermagem e entregues aos especialistas para realização dos diagnósticos de enfermagem, num instrumento a parte.

Os dados coletados do instrumento de pesquisa foram organizados em planilhas do software Microsoft Excel 2007 e do aplicativo SPSS versão 13.0, para avaliação da distribuição amostral dos diagnósticos de enfermagem (frequência simples e percentual), apresentada na forma de tabela. Na análise dos dados foram considerados os diagnósticos que obtiveram $50 \%$ de concordância entre os peritos.

Este estudo foi aprovado pelo Comitê de Ética em Pesquisa da Faculdade de Medicina do Hospital Universitário Antônio Pedro sob o no 293/09, atendendo aos preceitos da Resolução $n^{\circ} 466 / 12$ do Conselho Nacional de Saúde (CNS).

\section{RESULTADOS}

Dos 20 pacientes que participaram do estudo, a maioria era do sexo feminino (80\%), com idade entre 50 a 69 anos (75\%), ensino fundamental incompleto (60\%) e tinha a aposentadoria como principal fonte de renda (75\%).

Quanto aos fatores de risco, os mais prevalentes foram: veias varicosas (75\%), história familiar de doença venosa (45\%), longos períodos em pé ou sentado (40\%), trombose venosa profunda (35\%) e cirurgia venosa prévia (25\%).

Dentre as doenças de base encontradas pode-se destacar que as de maior evidência foram: insuficiência venosa crônica (100\%), hipertensão arterial sistêmica (65\%) e diabetes Mellitus (30\%).

Na Tabela 1 apresentam-se os 16 diagnósticos de enfermagem encontrados nos pacientes com úlcera venosa, conforme a classificação NANDA-I, agrupados segundo os oito domínios aos quais pertencem.

Tabela 1: Frequência dos diagnósticos de enfermagem identificados segundo domínios da NANDA-I em pacientes com úlcera venosa. Niterói/RJ, 2012

\begin{tabular}{cccc}
\hline Domínios & Diagnósticos de enfermagem & $\mathbf{N}$ & \% \\
\hline Promoção da saúde & Autocontrole ineficaz da saúde & 20 & 100 \\
& Volume de líquidos excessivo & 19 & 95 \\
Nutrição & Risco de volume de líquidos deficiente & 14 & 70 \\
& Nutrição desequilibrada mais do que as necessidades corporais & 14 & 70 \\
& Risco de glicemia instável & 6 & 30 \\
& Nutrição desequilibrada menos do que as necessidades & 1 & 5 \\
Atividade/Repouso & corporais & 20 & 100 \\
& Mobilidade física prejudicada & 100 \\
Autopercepção & Perfusão tissular periférica ineficaz & 30 \\
Papéis/Relacionamentos & Padrão de sono prejudicado & 1 & 5 \\
Enfrentamento/Tolerância ao & Déficit no autocuidado para banho & 15 \\
estresse & Distúrbio na imagem corporal & 75 \\
Segurança/Proteção & Interação social prejudicada & 20 \\
Conforto & Ansiedade & 13 & 65 \\
\hline
\end{tabular}




\section{DISCUSSÃO}

Dos 13 domínios da classificação NANDA-I houve prevalência de oito, estes relacionados tanto a padrões biofisiológicos como psicossociais. Os diagnósticos descritos reforçam a necessidade de deslocar a atenção dos profissionais de enfermagem dos aspectos físicos e biológicos da ferida e voltar seus olhares para o individuo em sua totalidade. Para tanto, é fundamental o uso de métodos capazes de fundamentar esse planejamento.

O principal diagnóstico de enfermagem para os pacientes com úlceras venosas foi Integridade tissular prejudicada. Um estudo mostra que os enfermeiros tem pesquisado muito mais sobre os diagnósticos de Integridade da pele prejudicada e Risco de integridade da pele prejudicada do que a respeito do diagnóstico Integridade tissular prejudicada, que representa o estágio mais avançado da ferida quando há dano as camadas da pele, atingindo tecidos mais profundos ${ }^{(11)}$.

O fato do diagnóstico Integridade tissular prejudicada aparecer pouco nos estudos de incidência e prevalência de diagnósticos de enfermagem, pode significar que o enfermeiro não tem identificado esse diagnóstico na clínica ou que ele está sendo diagnosticado, de forma mais branda, como Integridade da pele prejudicada e tratado como tal ${ }^{(11)}$. Novos estudos sobre validação e ocorrência desses diagnósticos têm sido realizados no Brasil e trarão resultados mais completos e atuais ${ }^{(7,9)}$.

Para estabelecer distinções entre os diagnósticos Integridade da pele prejudicada e Integridade tissular prejudicada, tais termos foram definidos a partir das camadas da epiderme. Ou seja, o paciente cujas lesões acometem os tecidos adiposo e muscular foram diagnosticados com Integridade tissular prejudicada. Em pesquisa com 42 pacientes que apresentavam úlceras vasculogênicas, o diagnóstico Integridade Tissular Prejudicada também foi entendido dessa maneira ${ }^{(9)}$.

Por se tratarem de úlceras venosas, o diagnóstico Perfusão tissular periférica ineficaz é relevante a esta clientela. A perfusão inadequada compromete todo processo de cicatrização, visto que a deficiência de oxigênio impede a síntese do colágeno, diminuindo a proliferação e migração celular e reduz a resistência dos tecidos à infecção. Logo, o tratamento dessas úlceras deve envolver medidas que auxiliem o retorno venoso e diminuam o edema ${ }^{(12-13)}$.

O edema, por sua vez, foi a principal característica clínica que indicou a presença do diagnóstico Volume de líquidos excessivo nesses pacientes. Em geral, é observado na região perimaleolar ou estende-se ao terço inferior da perna e está frequentemente associado à insuficiência venosa crônica. Devem ser empreendidas medidas para controle do edema, já que prejudica o fluxo sanguíneo e, com isso, retarda o processo de cicatrização das feridas, à medida que interfere na oxigenação e nutrição dos tecidos em formação ${ }^{(12,14)}$.

Duas técnicas eficazes para reduzir o edema são a drenagem linfática manual, cuja ação principal ocorre sobre o sistema linfático superficial, favorecendo a circulação sanguínea de retorno, e a compressão elástica, que age tanto na micro quanto na macrocirculação dos membros inferiores, o que diminui o refluxo patológico durante a deambulação e aumenta o volume de ejeção durante a ativação dos músculos da panturrilha ${ }^{(12,14)}$.

Além disso, cabe destacar que pacientes com feridas têm maior Risco de infecção. O estabelecimento de infecções nas feridas é um dos fatores responsáveis pelo retardo no processo de cicatrização. Nesse sentido, é conveniente definir que as feridas abertas podem estar colonizadas, quando verifica-se presença de microrganismos na ferida, sem invasão tecidual, ou, infectadas, quando os microrganismos invadem os tecidos da ferida, proliferando-se e provocando reações inflamatórias locais ${ }^{(15-16)}$.

Outro diagnóstico importante, advindo do retardo no processo de cicatrização das úlceras venosas e sua cronicidade, é Mobilidade física prejudicada, sobretudo em decorrência da dor crônica, do edema nos membros inferiores e da força muscular diminuída ${ }^{(10)}$.

A cronicidade da ferida associada às doenças de base e a falta de conhecimento dos pacientes sobre sua 
patologia são os fatores que comumente contribuem para o diagnóstico Autocontrole ineficaz da saúde, levando à recidivas frequentes. Nesse sentido, além de orientar o paciente quanto aos cuidados com a úlcera venosa, é necessário esclarecê-lo sobre sua doença de base e as atividades necessárias para o autocuidado, cabendo ao profissional atentar para a prevenção do surgimento de novas feridas ${ }^{(14,17)}$.

O diagnóstico de enfermagem Dor crônica refere-se a experiência sensorial e emocional desagradável associada a lesão tecidual real ou potencial, ou descrita em termos dessa lesão. Apresenta início súbito ou lento, de intensidade leve a intensa, constante ou recorrente, sem um término antecipado ou previsível e com uma duração de mais de seis meses ${ }^{(6)}$. Trata-se de uma das principais queixas de pessoas com feridas. Estima-se que, de cada 10 pessoas com úlceras crônicas, seis vivenciam a dor continuadamente ou não conseguem aliviá-la ${ }^{(18)}$.

Fatores como alterações no sono, mobilidade prejudicada, isolamento social, desequilíbrio econômico, desconforto físico e emocional estão comumente associados aos quadros de dor crônica ${ }^{(17,19)}$. O número significativo de pacientes com o diagnóstico Dor crônica mostra que a dor deve ser foco frequente da atenção do enfermeiro, o qual precisa estar atento para intervir adequadamente.

As circunstâncias de sofrimento vivenciadas por esses pacientes estão relacionadas também ao diagnóstico de enfermagem Distúrbio na imagem corporal, que reflete uma visão alterada do próprio corpo, o que faz com que estes pacientes evitem contatos sociais. As consequências desse isolamento incluem ansiedade e depressão ${ }^{(20)}$.

Em estudo realizado com 60 pacientes onde foi avaliado o nível de ocorrência de sintomas depressivos, 90\% deles apresentaram Distúrbio da imagem corporal, o que indica a necessidade de reavaliar efetividade da assistência prestada aos pacientes com feridas. Isto é, deve-se buscar alterações emocionais nesses pacientes, tanto em hospitais como em ambulatórios, para que seja possível intervir adequadamente ${ }^{(21)}$.

Outro diagnóstico de enfermagem relevante no processo de reparo dos tecidos, que foi pouco identificado, foi Nutrição desequilibrada: menos do que as necessidades corporais. Tal diagnóstico está relacionado ao aporte deficiente de nutrientes, quando os pacientes ingerem menos que as necessidades do organismo. Por outro lado, o diagnóstico Nutrição desequilibrada: mais do que as necessidades corporais foi prioritário na amostra.

O excesso de peso, e a obesidade estão associados ao retardo da cicatrização de feridas decorrente do comprometimento da circulação sanguínea e da hipoventilação que reduzem a perfusão de oxigênio e nutrientes nos tecidos ${ }^{(22)}$. Por isso, a avaliação nutricional deve ser um processo contínuo de obtenção e interpretação de dados com o propósito de determinar as melhores possibilidades de intervenção nutricional para o individuo. É importante levar em consideração não apenas os aspectos fisiopatológicos, mas também os fatores socioeconômicos, educacionais e psicoemocionais do paciente com úlcera venosa para planejar ações efetivas que possam proporcionar uma melhor qualidade de vida ${ }^{(23)}$.

O diagnóstico Risco de volume de líquidos deficiente foi identificado nos pacientes pelo ressecamento da pele, assim como pelos relatos sobre o baixo volume de líquido ingerido nas 24 horas do dia. Tais achados corroboram com a pesquisa realizada na Estratégia Saúde da Família do Espírito Santo, que observou ingestão de líquido abaixo da quantidade ideal para a manutenção da homeostasia em $73 \%$ dos idosos avaliados. A ingestão hídrica adequada deve ser ensinada a esses pacientes, pois favorece a absorção de nutrientes, hidratação e regeneração da pele ${ }^{(12)}$.

O diagnóstico de enfermagem Ansiedade foi identificado nos pacientes que relataram sensações de preocupação e angústia, durante a execução de atividades cotidianas. Trata-se de um achado coerente 
com as circunstâncias clínicas vividas por esses pacientes, dado a cronicidade da doença e o retardo na cicatrização, que por vezes arrasta-se há anos ${ }^{(20)}$.

$\mathrm{Na}$ amostra estudada observa-se correlação estreita entre os diagnósticos com maior incidência. Isto significa que ao investir na resolução/melhora de um dos diagnósticos, pode-se estimar a melhora dos resultados em geral.

Dessa forma, a equipe de enfermagem desempenha uma função primordial no cuidado à pessoa com úlcera venosa no que tange ao registro e identificação adequada dos diagnósticos de enfermagem, os quais, por sua vez, nortearão os cuidados de enfermagem a serem planejados e executados.

\section{CONCLUSÃO}

Ao analisar os diagnósticos de enfermagem em pacientes com úlcera venosa crônica em atendimento ambulatorial permitiu-se identificar 16 diagnósticos, distribuídos em oito domínios da NANDA-I. Os diagnósticos prevalentes em todos os pacientes foram: Integridade tissular prejudicada, Perfusão tissular periférica ineficaz, Risco de infecção, Mobilidade física prejudicada e Autocontrole ineficaz da saúde.

Os resultados deste estudo revelaram que os domínios Segurança/Proteção, Atividade/Repouso e Promoção da Saúde são fundamentais para orientar as intervenções de enfermagem, assim como, para avaliar os resultados referentes à prestação de cuidados possibilitando o uso seguro de uma linguagem padronizada.

Uma das limitações do estudo foi o tamanho da amostra, portanto, sugere-se que sejam feitas pesquisas com um número maior de participantes, para testar a acurácia dos diagnósticos que nortearão a prática clínica. De igual modo, recomenda-se o desenvolvimento de protocolos que tratem das intervenções e dos resultados de enfermagem em pacientes com úlceras crônicas.

\section{REFERÊNCIAS}

1. Dantas DV, Torres GV, Nóbrega WG, Macedo EAB, Costa IKF, Melo GSM et al. Assistência a portadores de úlceras venosas baseada em protocolos: revisão de literatura em bases de dados eletrônicas. Rev enferm UFPE [internet]. 2010 [cited 2015 mar 20]; 4(esp):1944-950. Available from:

http://www.revista.ufpe.br/revistaenfermagem/index.php/revista/arti cle/viewFile/1481/pdf_254.

2. Wong WW, Gurtner GC. Tissue engineering for the management of chronic wounds: current concepts and future perspectives. Exp Dermatol. 2012 [cited 2015 mar 19]; 21(10): 729-34. Available from: http://onlinelibrary.wiley.com/doi/10.1111/j.16000625.2012.01542.x/epdf.

3. Scotton MF, Miot HA, Abbade LPF. Factors that influence healing of chronic venous leg ulcers: a retrospective cohort. An. Bras. Dermatol. 2014 [cited 2015 mar 19]; 89(3): 414-422. Available from: http://dx.doi.org/10.1590/abd1806-4841.20142687.

4. Kieski G, Turra K. Sistematização da assistência de enfermagem na terapia compressiva: uma possibilidade terapêutica. Revista Eletrônica da Faculdade Evangélica do Paraná. 2012 [cited 2015 mar 21]; 2(4):1929. Available from:

http://www.fepar.edu.br/revistaeletronica/index.php/revfepar/article/ view/64/76.

5. North American Diagnosis Association - NANDA. Diagnósticos de enfermagem da NANDA: definições e classificações 2012-2014. Porto Alegre: Artmed; 2013.

6. Cavalcante AMRZ, Moreira A, Azevedo KB, Lima LR, Coimbra WKAM. Diagnóstico de enfermagem: integridade tissular prejudicada identificado em idosos na Estratégia de Saúde da Família. Rev. Eletr.

Enf. [Internet]. 2010 [citedo 2015 mar 25]; 12(4):727-35. Avaiable from: http://www.revistas.ufg.br/index.php/fen/article/view/8425. 7. Malaquias SG, Bachion MM, Nakatani AYK. Risco de integridade da pele prejudicada em idosos hospitalizados. Cogitare. Enferm. [Internet]. 2008 [cited 2015 mar 20]; 13(3):428-36. Avaiable from: http://132.248.9.34/hevila/Cogitareenfermagem/2008/vol13/no3/14. pdf.

8. Malaquias SG, Bachion MM, Martins MA, Nunes CAB, Torres GV, Pereira LV. Integridade tissular prejudicada, fatores relacionados e características definidoras em pessoas com úlceras vasculares. Texto Contexto Enferm [internet]. 2014 [cited 2015 mar 20]; 23(2): 434-42. Available from: http://www.redalyc.org/pdf/714/71431352025.pdf. 9. Lima MSFS, Carvalho ESS, Silva EA, Gomes WS, Passos SSS et AL. Diagnósticos de enfermagem evidenciados em mulheres com feridas crônicas. Rev baiana enferm. [Internet]. 2012 [cited 2015 mar 20]; 26(3):585-92. Avaiable from:

http://www.portalseer.ufba.br/index.php/enfermagem/article/viewAr ticle/6740.

10. Ribeiro MAS, Lages JSS, Lopes MHBM. Diagnósticos de enfermagem relacionados a pele: definições operacionais. Rev. Latino-Am. Enfermagem. [internet]. 2012 [cited 2015 mar 19];20(5):1-10. Available from: http://www.scielo.br/pdf/rlae/v20n5/pt_07.pdf.

11. Azoubel R, Torres GV, Silva LWS, Gomes FV, Reis LA. Efeitos da terapia física descongestiva na cicatrização de úlceras venosas Rev. Esc. Enf. USP. [internet]. 2010 [cited 2015 mar 21]; 44(4): 1085-92. Available from: http://www.scielo.br/pdf/reeusp/v44n4/33.pdf. 12. Gomes T, Cade NV, Rohr RV, Fejoli MM. Caracterização das lesões crônicas e os fatores associados em moradores de um território de saúde em Vitória, Espírito Santo. Rev. Bras. Pesqui. Saúde [internet]. 
2011 [cited 2015 mar 21]; 13(1): 52-57. Available from:

http://periodicos.ufes.br/RBPS/article/viewFile/1330/991.

13. Abreu AM, Oliveira BRB, Manarte JJ. Treatment of venous ulcers with an unna boot: a case study. Online Braz J Nurs [Internet]. 2013 [cited 2015 mar 22]; 12 (1): 198-208. Available from:

http://www.objnursing.uff.br/index.php/nursing/article/view/3845. 14. Santos SLV, Martins MA, Vasconcelos LSNOL, Lima ABM , Malaquas SG, Maria Márcia Bachion MM. Bastonetes gram-negativos em úlceras venosas e implicações para o atendimento de enfermagem na atenção primária. Rev. Eletr. Enf. [internet]. 2014 [cited 2015 mar 21]; 16(2):370-7. Available from:

http://www.fen.ufg.br/fen_revista/v16/n2/pdf/v16n2a13.pdf.

15. Greer N, Foman N, Dorrian J, Fitzgerald P, Macdonald R, Rutksi I, Wilt Timothy. Advanced wound care therapies for non-healingdiabetic, venous, and arterial ulcers: a systematic review. Washington: department of veterans affairs [internet]. 2012 [cited 2015 mar 22]. Available from:

http://www.ncbi.nlm.nih.gov/pubmedhealth/pmh0054957.

16. Malaquias SG, Bachion MM, Santana SMSC, Dallarmi CCB, Lino Junior RS, Ferreira PS. Pessoas com úlceras vasculogênicas em atendimento ambulatorial de enfermagem: estudo das variáveis clínicas e sociodemográficas. Rev. Esc. Enf. USP [internet]. 2012 [cited 2015 mar 22]; 46(2): 302-10. Available from:

http://www.scielo.br/pdf/reeusp/v46n2/a06v46n2.pdf.

17. Macedo EAB, Oliveira AKA, Melo GSM, Nobrega WG, Costa IKF, Dantas DV, et al. Characterization sociodemographic of patients with venous ulcers treated at a university hospital. Rev enferm UFPE [internet]. 2010 [cited 2015 mar 23]; 4 (spe):1919-963. Available from: http://www.revista.ufpe.br/revistaenfermagem/index.php/revista/arti cle/viewArticle/1475.

18. Calasans MT, Amaral JB, Carvalho ESS. O manejo da dor em pessoas que vivem com feridas. In: Carvalho ESS, editor. Como cuidar de pessoas com feridas: desafios para prática multiprofissional. Salvador: Atualiza; 2012. p.293-316.

19. Cunha L, Mayrink WC. Influencia da dor crônica na qualidade de vida em idosos. Rev. Dor [internet]. 2011 [cited 2015 mar 23]; 12(2):120- 4. Available from: http://www.scielo.br/pdf/rdor/v12n2/v12n2a08.

20. Waidman MAP, Rocha SC, Correa JL, Brischiliari A, Marcon SS. O cotidiano do indivíduo com ferida crônica e sua saúde mental. Texto Contexto Enferm [internet]. 2011 [cited 2015 mar 23]; 20(4): 691-9. Available from: http://www.scielo.br/pdf/tce/v20n4/07.pdf. 21. Salome GM, Blanes L, Ferreira LM. Avaliação de sintomas depressivos em pessoas com úlcera venosa. Rev. Bras. Cir. Plást. [internet]. 2012 [cited 2015 mar 20]; 27(1):124-9. Available from: http://www.scielo.br/pdf/rbcp/v27n1/21.pdf.

22. Perrone F, Paiva AA, Letícia Souza LMI, Faria CS, Paese MCS, Nascimento JEA et al. Estado nutricional e capacidade funcional na úlcera por pressão em pacientes hospitalizados. Rev. Nutr. [internet]. 2011[cited 2015 mar 21]; 24(3): 431-43. Available from:

http://www.scielo.br/scielo.php?script=sci_arttext\&pid=S1415$\underline{52732011000300006 .}$.

23. Posthauer ME, Doner B, Collins N. Nutrition: a critical component of wound healing. Adv Skin Wound Care [internet]. 2010 [cited 2015 mar 23]; 23(2):560-72. Available from:

http://www.ncbi.nlm.nih.gov/pubmed/21084879.

Recebido: 07/03/2014.

Aceito: 04/03/2015.

Publicado: 30/06/2015. 